\title{
Anisotropic Quadrilateral Mesh Generation: An Indirect Approach
}

\author{
D. Merhof ${ }^{a, *}$, R. Grosso ${ }^{a}$ and U. Tremel ${ }^{b}$ G. Greiner ${ }^{a}$ \\ ${ }^{a}$ Computer Graphics Group, Friedrich-Alexander University of \\ Erlangen-Nuremberg, Am Weichselgarten 9, 91058 Erlangen, Germany \\ ${ }^{\mathrm{b}}$ EADS Military Aircraft, Flight Physics / Numerical Simulation - MT632, \\ 81663 Munich, Germany
}

\begin{abstract}
In this paper a new indirect approach is presented for anisotropic quadrilateral mesh generation based on discrete surfaces. The ability to generate grids automatically had a pervasive influence on many application areas in particularly in the field of Computational Fluid Dynamics. In spite of considerable advances in automatic grid generation there is still potential for better performance and higher element quality. The aim is to generate meshes with less elements which fit some anisotropy criterion to satisfy numerical accuracy while reducing processing times remarkably. The generation of high quality volume meshes using an advancing front algorithm relies heavily on a well designed surface mesh. For this reason this paper presents a new technique for the generation of high quality surface meshes containing a significantly reduced number of elements. This is achieved by creating quadrilateral meshes that include anisotropic elements along a source of anisotropy.
\end{abstract}

Key words: mesh generation, surface mesh, quadrilateral, anisotropy, unstructured meshes, advancing front approach

\section{Introduction}

The generation of surface meshes has been a widely explored topic. Algorithms for the generation of triangular surface meshes are currently used in many different industrial and research applications. Recently, attention has also been

* Corresponding author. 
given to the generation of quadrilateral surface meshes $(1 ; 2 ; 3 ; 4)$. The reason is that for a given node density, quadrilateral meshes contain only half as many elements as triangular meshes. The saving of elements continues when a volume mesh is generated using, for example, an advancing front method. If quadrilateral meshes serve as starting point for the volume mesh creation, the resulting hexahedral meshes provide the same quality (node density) as the equivalent tetrahedral mesh derived from a triangular surface but comprise a significantly lower number of volume elements, which is a highly desirable characteristic. For reasons of better accuracy and efficiency, many applications prefer quadrilateral elements for two-dimensional meshes and hexahedral elements for three-dimensional meshes $(5 ; 6)$.

A second possibility to reduce the number of surface elements, and as a result the number of volume elements, is to introduce anisotropic elements. Anisotropic meshes have the property that the density of nodes is direction dependent. Several previous attempts have been made for developing algorithms to gain anisotropic triangular meshes $(7 ; 8 ; 9 ; 10 ; 11)$. Lee $(12)$ presented a method to transform these meshes into quadrilateral meshes preserving the anisotropic characteristic of the triangular mesh.

The goal of this paper is to extend the benefits of unstructured quadrilateral meshes by including anisotropic elements in indirect mesh generation. Contrary to the approach presented by Lee (12), our algorithm works on isotropic triangular meshes and incorporates anisotropic quadrilaterals along a line source of anisotropy defined by the user. Thereby, quasi-structured rows of anisotropic quadrilaterals are generated along the source. This approach provides the possibility to generate application optimized quadrilateral meshes with a significantly reduced number of elements. Based on these surface meshes, hexahedral meshes may be generated according to the approaches described by $(13 ; 14 ; 15)$.

The paper is organized as follows: In Section 2, a smoothing approach for surface meshes in 3D is presented. The method is based on a local Least Square fit of the surface. In Section 3, the techniques for the generation of anisotropic quadrilaterals along a source of anisotropy are described. Finally, an example is presented demonstrating the benefits of our approach. Possible future work is also discussed.

\section{Method}

The algorithm for anisotropic quadrilateral mesh generation presented in this paper works on high resolution triangular meshes in 3D and is completely independent of an analytic surface description. This is important especially 
when only legacy triangular meshes are available. The initial triangular meshes are the base for the anisotropic quadrilateral mesh generation. In addition, the user has to specify a source of anisotropy and a maximum anisotropy. The source of anisotropy is given through a subset of initial front edges connected to a line. Along the anisotropy source, quadrilateral elements with maximum anisotropy will be found. With growing distance to the source the anisotropy decreases depending on the mesh size of the underlying triangular mesh.

For reasons of unrestricted applicability the algorithm was extended to curved surfaces in 3D. For this purpose a local surface approximation was implemented to enable smoothing during three-dimensional surface mesh generation. Smoothing operations may then be performed by moving nodes on the approximated surface. Well established smoothing techniques commonly used are designed for two-dimensional meshes, for this reason they had to be adapted to three dimensions and combined with the surface approximation.

This paper therefore comprises two major contributions:

- An algorithm for the indirect generation of anisotropic quadrilateral meshes and

- an approach for smoothing of arbitrary three-dimensional surface meshes.

In this section we continue with the presentation of the techniques developed for smoothing of three-dimensional meshes. In Section 3, the algorithm for the generation of anisotropic quadrilaterals is presented.

\subsection{Surface Approximation}

The presented approach for local surface approximation provides the possibility to process three-dimensional surface meshes completely independent of analytic CAD data. This is of special importance in case of legacy triangular meshes where a parametric representation is not available. Löhner (16) designates a number of situations where this occurs.

The surface approximation is used to perform smoothing operations, i.e. to move nodes on the approximated surface. For each node that has to be smoothed, an analytic surface is determined by the following conditions: (a) The surface intersects the given node and (b) the surface is a best (Least Squares) fit to the fan of neighbor nodes around the given node, i.e. all direct neighbor nodes are as close to the surface as possible. The analytic surface is locally approximated 
by a biquadratic Taylor polynomial (where $u, v$ are the $2 \mathrm{D}$ parametric coordinates of each node relative to the center node positioned at $\left.\left(u_{s}, v_{s}\right)=(0,0)\right)$ :

$$
F(u, v)=u F_{u}+v F_{v}+\frac{u^{2}}{2} F_{u u}+u v F_{u v}+\frac{v^{2}}{2} F_{v v}
$$

where the coefficients $F_{u}, F_{v}, F_{u u}, F_{u v}$ and $F_{v v}$ are the unknowns. They are actually the derivatives of the biquadratic polynomial. With the help of an exponential projection the 3D position of all neighbor nodes is transformed into 2D parameter space. Afterwards, the coefficients of the biquadratic form are computed using a Least Squares approach by solving an appropriate system of linear equations. The required computations and processing steps are given in detail in Section 2.1.1 and 2.1.2. The analytic surface approximation is essential for performing smoothing operations in $3 \mathrm{D}$.

\subsubsection{D Parameter Space Coordinates}

Smoothing of a node $N_{s}$ is performed by moving the node on an analytic surface that runs through the node itself and best fits all surrounding neighbor nodes connected to $N_{s}$ by an edge. For a local surface approximation around $N_{s}$ the first and second partial derivatives of the approximating biquadratic polynomial must be determined. To compute the derivatives $F_{u}, F_{v}, F_{u u}, F_{u v}$ and $F_{v v}$, an almost isometric parameterization $F\left(u_{i}, v_{i}\right)=N_{i}$ of the neighborhood of $N_{s}$ with $N_{s}=(0,0,0)=F(0,0)=F\left(u_{s}, v_{s}\right)$ is needed. A parameterization is thereby called isometric if $\left\|F_{u}\right\| \approx 1,\left\|F_{v}\right\| \approx 1$ and $F_{u} F_{v} \approx 0$.

The exponential projection that was selected as parameterization takes length and angle of neighbor edges into account. Furthermore, the order of the nodes around the center node is maintained. For every neighbor node $N_{i}$ the coordinates $\left(u_{i}, v_{i}\right)$ in $2 \mathrm{D}$ parameter space are determined using the following exponential projection:

$$
\exp \left(N_{i}\right) \mapsto\left\|N_{i}\right\|\left(\cos \left(\sum_{j=1}^{i-1} \tilde{\alpha}_{j}\right), \sin \left(\sum_{j=1}^{i-1} \tilde{\alpha}_{j}\right)\right)
$$

where $N_{i}$ are the coordinates of node $i$ in 3D and $\alpha_{i}$ is the angle between the neighbor nodes $N_{i}, N_{i+1}$. The angles $\tilde{\alpha}_{i}$ are thereby computed by a function flat that scales the angles $\alpha_{i}$ so that the sum of the projected angles $\tilde{\alpha}_{i}$ amounts to $2 \pi$ (Figure 1 ). These conditions together with the projection of the central node $N_{s}$ uniquely define a parameterization $F\left(\exp \left(N_{i}\right)\right)=N_{i}$. Do Carmo (17) showed that this is a local isometric parameterization. 


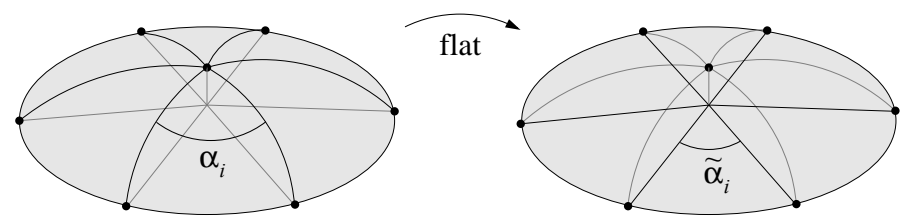

Fig. 1. Function flat scales the angles $\alpha_{i}$ between two neighbor nodes $N_{i}$ and $N_{i+1}$. Resulting angles $\tilde{\alpha}_{i}$ amount to $2 \pi$.

Regarding the choice of $f l a t$, there are several possibilities. The current implementation uses uniform scaling:

$$
\tilde{\alpha}_{i}=\operatorname{flat}\left(\alpha_{i}\right)=\alpha_{i} \frac{2 \pi}{\sum_{j} \alpha_{j}}
$$

This definition of flat scales the angles in a uniform manner so that the sum of angles $\tilde{\alpha}_{i}$ amounts to $2 \pi$. This approach works for any configuration of neighbors around a node and maintains the order of neighbor nodes around the center node (18).

\subsubsection{Computation of Derivatives}

After having determined a parameterization by computing the exponential projection described above, the derivatives $F_{u}, F_{v}, F_{u u}, F_{u v}$ and $F_{v v}$ are obtained by solving a linear system of equations

$$
\mathbf{V} \vec{F}=\mathbf{N} \text {. }
$$

The rows of matrix $\mathbf{V}$ contain for each neighbor node $i$ the coefficients $\left(u_{i}, v_{i}, \frac{u_{i}^{2}}{2}, u_{i} v_{i}, \frac{v_{i}^{2}}{2}\right)$. The matrix $\mathbf{N}$ contains the $3 \mathrm{D}$ coordinates $N_{i}$ of all neighbor nodes. After solving the system of equations the vector $\vec{F}$ contains the derivatives, i.e. $\vec{F}=\left(F_{u}, F_{v}, F_{u u}, F_{u v}, F_{v v}\right)^{T}$. The solution of the system of equations is computed using a Least Squares or Least Norm approach:

$$
\vec{F}=\left\{\begin{array}{r}
\mathbf{V}^{T}\left(\mathbf{V} \mathbf{V}^{T}\right)^{-1} \mathbf{N}: n<5 \\
\mathbf{V}^{-1} \mathbf{N}: n=5 \\
\left(\mathbf{V}^{T} \mathbf{V}\right)^{-1} \mathbf{V}^{T} \mathbf{N}: n>5
\end{array}\right.
$$

where $n$ is the total number of neighbor nodes $N_{i}$. A formal proof for issues like invertability and numerical stability is not given at this point. However, 
depending on the number of neighbor nodes, several conditions for invertability of the respective matrix are available:

- If the center node is surrounded by three neighbor nodes, the matrix is invertible if the neighbor nodes are not identical.

- In case of four neighbor nodes, the matrix is invertible if the neighbor nodes are not identical and do not lie on a line which is rather improbable and in general not possible in a valid mesh.

- If the number of neighbor nodes amounts to five, the matrix is invertible if the neighbor nodes are not identical and if $N_{s}$ located at the origin does not lie on a quadric defined by the neighbor nodes. In general, a quadric is uniquely defined by five points in a plane. Again, the case that the center node also lies on the quadric is very improbable and also not possible in a valid mesh.

These conditions are derived by considering determinants of the respective matrix. If no solution is found, the smoothing process is aborted and the center node $N_{s}$ remains at its original position. However, an empirical observation is that these cases are so improbable or even impossible in a valid mesh that the smoothing procedure always succeeded so far.

The solution of the system of equations provides the derivatives $F_{u}, F_{v}, F_{u u}, F_{u v}$ and $F_{v v}$ of the biquadratic Taylor polynomial. After smoothing in 2D parameter space this polynomial allows to reposition nodes on the analytic surface in $3 \mathrm{D}$ space. The following section gives a short overview of how this local surface fit is used for smoothing of surface meshes in 3D.

\subsection{D Smoothing}

For smoothing of two-dimensional quadrilateral meshes there exists a number of well-established techniques $(1 ; 2 ; 20)$. A selection of $2 \mathrm{D}$ smoothing techniques were adapted to $3 \mathrm{D}$ by combining them with the local surface approximation presented above. The current implementation of 3D smoothing uses Laplacian, length, angle and isoparametric smoothing in combination with the local surface fit.

A problem arising with the surface approximation approach is that the transformation from 3D space to 2D parameter space by applying the exponential projection does not preserve angles or lengths. For this reason the smoothing operations had to be modified and adjusted if necessary. Furthermore, decisions had to be made which part of the smoothing operation has to be performed in $3 \mathrm{D}$ or in $2 \mathrm{D}$, i.e. it has to be considered if it is more convenient to perform the smoothing operation before the exponential projection or afterwards. Once a node movement has been carried out in $2 \mathrm{D}$, the new node 
position in 3D is easily computed using the local surface fit, thus preserving the geometric shape of curved surfaces. The accuracy of the approximation is limited by the underlying triangular mesh which is assumed to be of high resolution. In Figure 2 the quality of the 3D smoothing approach based on the local surface approximation is demonstrated.
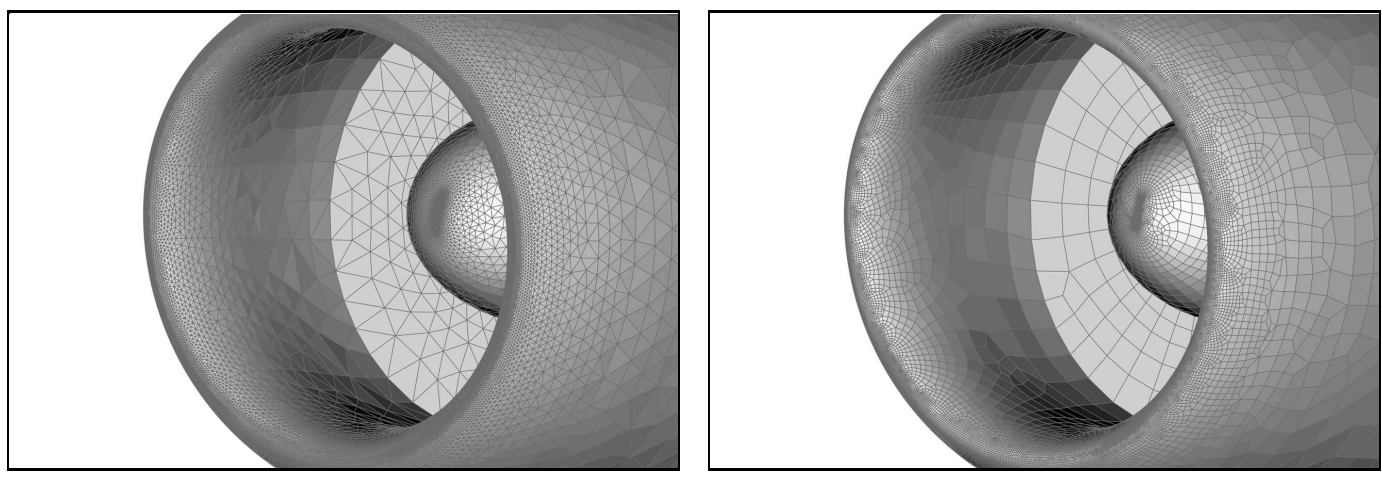

Fig. 2. Air plane engine, smoothing of three-dimensional surface. Left: Triangular mesh. Right: Quadrilateral mesh, smoothing with local surface approximation.

Since triangular meshes usually resolve geometric detail with an appropriate resolution of the mesh, i.e. the resolution of the mesh is sufficiently high, in most cases this approach provides adequate exactness. The divergence between original triangular mesh and quadrilateral mesh was investigated in (19) by measuring Hausdorff distances. These measurements showed that the shape of the objects is well preserved even after repeated smoothing passes during quadrilateral mesh generation.

\section{Anisotropic Quadrilateral Meshes}

The indirect generation of quadrilateral surface meshes reduces the number of mesh elements to approximately fifty percent while keeping the original node density which stands for quality of resolution. The number of elements can be further reduced by introducing anisotropic elements. For a fluid flow computation anisotropic quadrilaterals may be used at parts of the mesh where a lower resolution in one direction has no effect on the quality of the flow computation, e.g. along the leading edge of an air plane wing. For the generation of an anisotropic quadrilateral mesh the following steps are performed:

- The source of anisotropy is segmented according to the maximum anisotropy that is required.

- Along the anisotropy source quasi-structured rows of isotropic quadrilaterals are created. 
- These quadrilaterals are merged to obtain anisotropic quadrilaterals.

- A connection between the anisotropic quadrilaterals and the remaining triangular mesh is established.

- The remaining triangular mesh is filled with isotropic quadrilaterals.

One major contribution of this work is an advancing front algorithm for the generation of quasi-structured rows of isotropic quadrilaterals aligned to the line source of anisotropy which are suitable for anisotropic merging. The remaining triangular mesh is transformed into an isotropic quadrilateral mesh using our implementation of the Q-Morph algorithm (2). Both algorithms make use of the surface approximation described in Section 2 which is essential for the required smoothing operations. The steps that are necessary for anisotropic quadrilateral mesh generation are described in more detail in the following sections.

\subsection{Source of Anisotropy}

A source of anisotropy that serves as origin of anisotropic elements has to be specified by the user. Any series of initial front edges connected to each other may be chosen as anisotropy source as long as the edges form a line. The maximum anisotropy has to be specified by the user, too. The aim is to have a maximum anisotropy at the source that decreases with growing distance to the source depending on the actual mesh size of the underlying triangular mesh.

\subsection{Anisotropic Quadrilaterals}

Anisotropic elements are generated using a novel approach which we called Three-Step-Method. Starting with a triangular mesh, first isotropic quadrilaterals are created in a structured manner (step one). The anisotropic elements are obtained by merging isotropic quadrilaterals (step two) and are connected
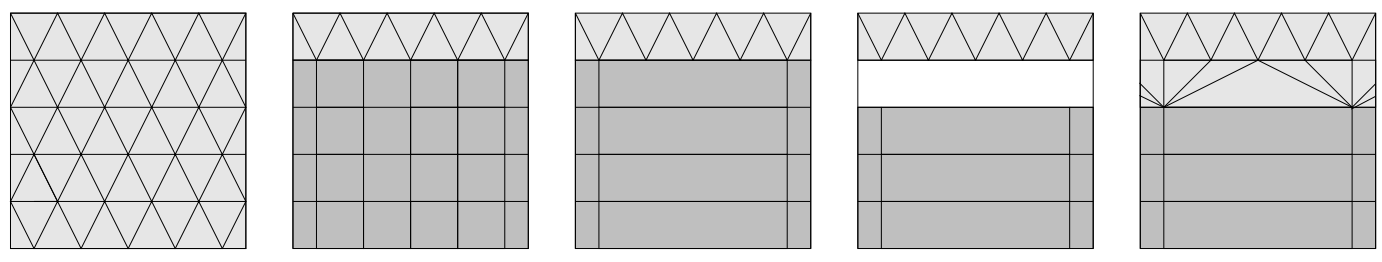

Fig. 3. Three-Step-Method. Left to right: Triangular mesh. Structured rows of isotropic quadrilaterals. Anisotropic merge of quadrilaterals. Deletion of upmost row of anisotropic quadrilaterals. Transition layer to triangular mesh. 
to the remaining triangular mesh (step three). The outlined three steps are shown in Figure 3.

In more detail, the generation of anisotropic elements works as follows: The line source of anisotropy is treated as initial front and several structured rows of isotropic quadrilaterals are generated. The algorithm for the generation of quadrilaterals for anisotropic merging thereby enforces the generation of structured rows aligned to the boundary. Otherwise complications would occur when anisotropic merging is performed. The source is then segmented, i.e. it has to be specified which source edges will be replaced by a single anisotropic edge. At the beginning of the source and the end of the source anisotropy slowly increases and decreases to avoid sudden changes in element size. Figure 4 shows a segmented source for a maximum anisotropy of 1:3.

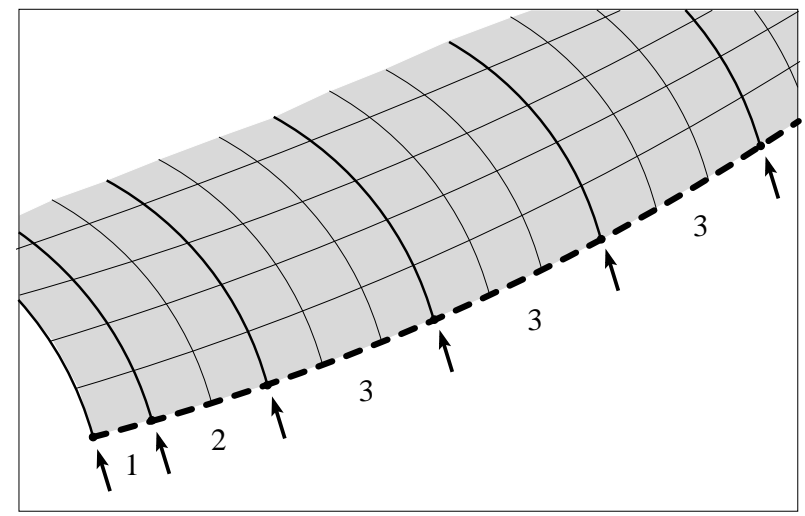

Fig. 4. Arrows indicate segmentation of anisotropy source (dashed line) for a maximum anisotropy of 1:3.

The isotropic structured quadrilaterals are then merged according to the segmentation of the source to obtain anisotropic quadrilaterals. The uppermost row of isotropic quadrilaterals is replaced by triangles that provide a transition from the anisotropic quadrilaterals to the remaining triangular mesh. This is necessary to keep the mesh closed and to avoid hanging nodes. After having transformed all structured rows of isotropic quadrilaterals into anisotropic quadrilaterals, the remaining triangular mesh is filled with isotropic quadrilaterals.

This approach has two limitations: The wing of an airplane typically has a high curvature at the leading edge leading to a dense sampling of surface points and therefore a high number of triangular elements. Contrary, the more planar parts of the wing are modeled with large triangles. This variation in element size restricts the number of structured rows that can be generated starting at the leading edge chosen as anisotropy source. A second limitation is that the maximum anisotropy that can be achieved is $1: 3$ or $1: 4$. Otherwise, the 
transition from the anisotropic to the isotropic area would produce neighboring elements that differ significantly in size as can be seen in Figure 5. As flow computations require a smooth transition of size between neighboring mesh elements, this is an undesirable feature. To overcome these limitations the method was extended which is described below.

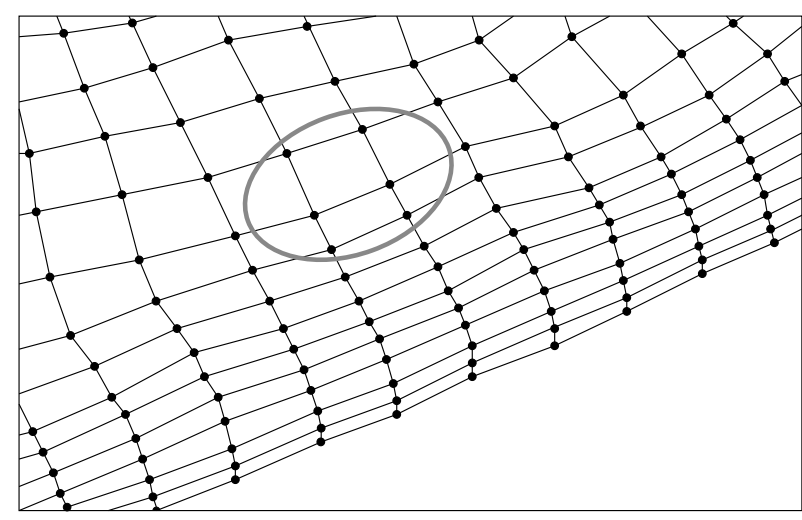

Fig. 5. Sudden transition from anisotropic to isotropic area appears in combination with higher anisotropy.

\subsection{Multi-Pass Approach}

The multi-pass approach provides more flexibility with regard to the number of anisotropic rows and allows a much higher anisotropy at the source. With a growing distance to the source the anisotropy declines according to the current mesh size of the underlying triangular elements. This is achieved by applying the Three-Step-Method several times (Multi-Pass Three-Step-Method): The first pass is identical to the Three-Step-Method described above, i.e. the source of anisotropy is used as initial front, a number of structured rows of isotropic quadrilaterals is generated and the isotropic quadrilaterals are merged to obtain anisotropic quadrilaterals. The second and all further passes operate in the same way with one essential difference: The top edges of the last row of anisotropic quadrilaterals are used as new initial front. This guarantees that the edge length fits better the actual edge size within the mesh so that further structured rows can be generated. However, the merging of quadrilaterals starts for each pass at the anisotropy source, i.e. all quadrilateral rows existing at this point receive a higher anisotropy. The final anisotropy at the source is hence multiplicative and is obtained by computing the product of the anisotropy relations of each pass (Figure 6). Each pass may have a different anisotropy but the last pass should have a small anisotropy, i.e. 1:2, to obtain a fluent transition to the remaining isotropic area. 

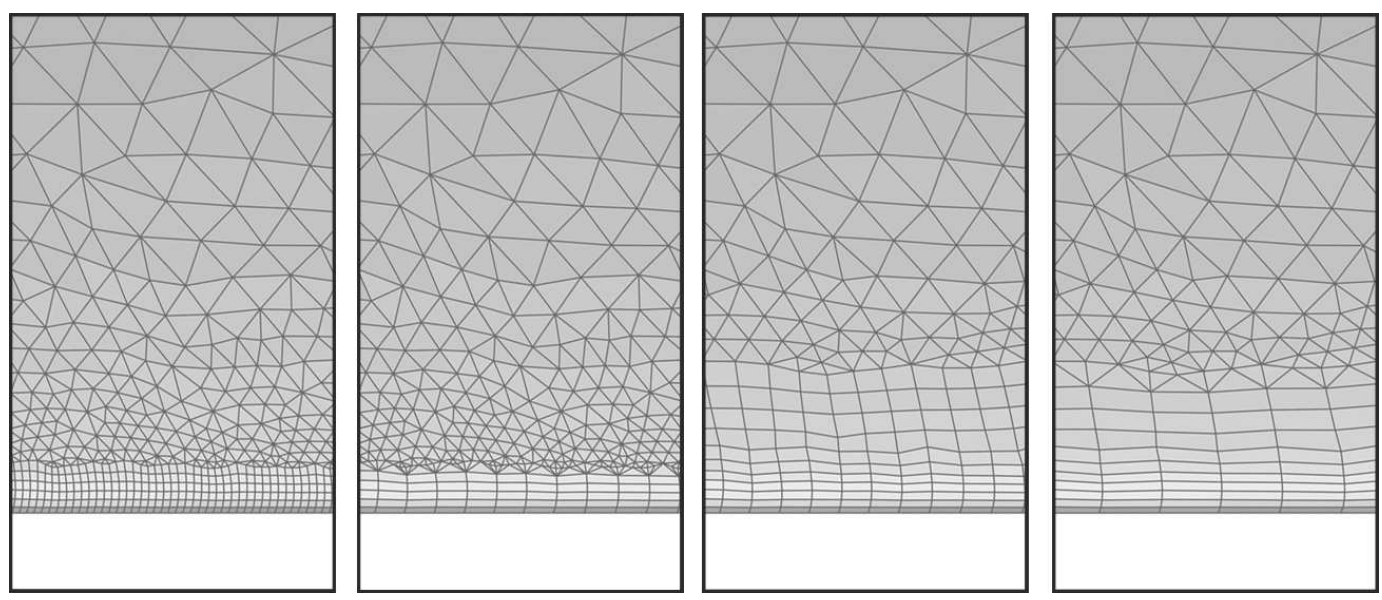

Fig. 6. Multi-Pass Three-Step-Method. Two left Figures: First pass, anisotropy 1:4. Two right Figures: Second pass, anisotropy 1:2. Resulting anisotropy at source: 1:8.

\subsection{Isotropic quadrilaterals}

As soon as the generation of structured rows of anisotropic quadrilaterals is accomplished, the remaining triangular mesh is transformed into an isotropic quadrilateral mesh using an implementation of the Q-Morph algorithm (2). The algorithm carefully converts and combines triangles of the background mesh to obtain quadrilateral elements. Starting with an initial front enclosing the domain to be gridded, the algorithm proceeds into interior regions by constantly generating new quadrilaterals. For the creation of each new quadrilateral, the following processing steps are performed: The algorithm selects an advancing front edge and chooses side edges from the mesh by considering angle criterions. The top edge is then created using swap operations if necessary. The triangles within this new quadrilateral are removed from the mesh, the new element consisting of the processed advancing front edge, the side edges and the top edge is inserted into the mesh and the advancing front is updated. These operations are repeated until the whole domain is covered with quadrilaterals. Thereby, a precondition for an all-quadrilateral mesh is an even number of initial front edges which has to be considered when patches are defined (otherwise, a single triangle remains).

\section{Results}

The quadrilateral mesh generator was tested with a series of triangular meshes and proved to be capable to handle quite different geometries. With regard to anisotropic elements, the focus was on the air plane application area where 
anisotropic elements along the leading edge of a wing are highly desirable. The reason is that along the leading edge the air flow changes little, thus a coarse sampling is sufficient. Contrary, strong air flow gradients are observed when following the air flow across the wing. These are optimal preconditions to introduce anisotropic quadrilaterals. Regarding the geometry of an air plane wing, a high curvature of the surface is observed perpendicular to the leading edge. This leads to a high number of surface elements in an isotropic mesh. By introducing anisotropic quadrilaterals along the leading edge, the quality of the flow computation is maintained but the number of surface elements is reduced dramatically, which is a highly desirable characteristic.

Figure 7 shows a wing example, the leading edge was chosen as anisotropy source. The Multi-Pass Three-Step-Method is applied using three passes with anisotropy 1:4, 1:2 and again 1:2. The first pass is shown in the first row: Beginning at the source of anisotropy, structured rows of isotropic quadrilaterals are created. Subsequently the isotropic quadrilaterals are merged to anisotropic quadrilaterals using an anisotropy of 1:4. The next pass starts from the top of the first pass. Again, structured rows of quadrilaterals are created. The anisotropic merging of elements with anisotropy 1:2 starts at the source, so the anisotropy at the source is 1:8 after pass two. The third pass produces a final anisotropy of 1:16 at the source while supplying a smooth 1:2 transition to the remaining triangular mesh. The last row shows the completed wing.

The reduction of elements achieved by using anisotropic quadrilaterals is remarkable: A mesh containing quadrilaterals instead of triangles only has about half as much elements. Introducing anisotropic quadrilaterals again lowers the number of elements significantly so that in the end less than twenty percent of elements remain.

Table 1 shows the reduction of elements within a quadrilateral mesh depending on the degree of anisotropy that was applied. Anisotropy further reduces the number of elements. Meshes with anisotropy 1:2 to 1:5 were created using the Three-Step-Approach, for all other cases a Multi-Pass Three-Step-Approach was applied.

\begin{tabular}{|l||c|c|c|c|c|c|c|c|}
\hline Anisotropy & $1: 1$ & $1: 2$ & $1: 3$ & $1: 4$ & $1: 5$ & $1: 6$ & $1: 8$ & $1: 16$ \\
\hline No. of quads & 4540 & 3327 & 2793 & 2452 & 2202 & 2064 & 1915 & 1658 \\
\hline$\%$ & 100 & 73 & 62 & 54 & 49 & 45 & 42 & 37 \\
\hline
\end{tabular}

Table 1

Depending on the degree of anisotropy the number of quadrilaterals is reduced significantly by using anisotropic elements. The original triangular mesh contained 8802 elements. 
These results are of major interest for the air plane industry. As flow computations for air planes last up to several days (in transient cases even weeks), there is urgent need for application optimized meshes such as the anisotropic quadrilateral meshes generated with the algorithm presented in this work.

\section{Conclusions and Future Work}

In this paper a new indirect approach for anisotropic quadrilateral mesh generation was presented. The quadrilateral generator was equipped with 3D smoothing techniques that provide the possibility to process three-dimensional triangular meshes. The local surface approximation introduced for this purpose is well suited to maintain the geometry of curved surfaces. The Multi-Pass Three-Step-Method developed for the creation of anisotropic quadrilaterals provides a smooth transition from anisotropic to isotropic parts of the mesh. Using a multi-pass approach a much higher anisotropy may be achieved than with a single-pass Three-Step-Method. The presented approach is of special interest for the air plane industry where anisotropic quadrilaterals along the leading edge of an air plane wing are highly desirable. The presented example demonstrates that the number of mesh elements is reduced considerably by including anisotropic quadrilaterals.

Future work will include the development of more sophisticated smoothing techniques that are optimized for anisotropic quadrilaterals. The creation of hexahedral boundary layers for anisotropic volume meshes will also be of interest as well as the parallelization of the techniques developed in this context.

\section{References}

[1] Blacker, T.D., Stephenson, M.B., "Paving: A new Approach to Automated Quadrilateral Mesh Generation", Int. J. for Numer. Meth. in Eng., 32, 811-847, 1991.

[2] Owen, S.J., Staten, M.L., Canann, S.A., Saigal, S., "Q-MORPH: an indirect approach to advancing front quad meshing”, Int. J. for Numer. Meth. in Eng., 44, 1317-1340, 1999.

[3] Shimada, K., Liao, J.H., Itoh, T., "Quadrilateral meshin gwith directionality control by packing square cells", In Proc. of 7th Int. Meshing Roundtable, 61-75, 1998.

[4] Borouchaki, H., Frey, P.J., "Adaptive triangular-quadrilateral mesh generation", Int. J. for Numer. Meth. in Eng., 41, 915-934, 1998.

[5] Brauer, J.R., "What every engineer should know about finite element analysis", Marcel Decker Inc., 1993. 
[6] Zienkiewicz, O.C., Taylor, R.L., "The finite element method", McGraw Hill, London, 1989.

[7] Bossen, F.J., Heckbert, P.S., "A Pliant Method for Anisotropic Mesh Generation", In Proc. of 5th Int. Meshing Roundtable, 63-74, 1996.

[8] Shimada, K., Yamada, A., Itoh, T., "Anisotropic Triangular Meshing of Parametric Surfaces via Close Packing of Ellipsoidal Bubbles", In Proc. of 6th Int. Meshing Roundtable, 375-390, 1997.

[9] Pirzadeh, S., "Unstructured Viscous Grid Generation by AdvancingLayers Method", AIAA-93-3453-CP, AIAA, 420-434, 1993.

[10] Borouchaki, H., Castro-Diaz, M.J., George, P.L., Hecht, F., Mohammadi, B., "Anisotropic adaptive mesh generation in two dimensions for CFD", Proc. 5th International Conference On Numerical Grid Generation in Computational Field Simulations, Vol 3, pp.197-206, 1996.

[11] George, P.-L., Borouchaki, H., "Delaunay Triangulation and Meshing: Application to Finite Elements", Hermes, 1998.

[12] Lee, Y.K., Lee, C.K., "Automatic generation of anisotropic quadrilateral meshes on three-dimensional surfaces using metric specifications", Int. J. for Numer. Meth. in Eng., 53, 2673-2700, 2002.

[13] Müller-Hannemann, M., "Improving the surface cycle structure for hexahedral mesh generation", Proc. 16th Symp. Computational Geometry, 19-28, 2000.

[14] Tautges, T.J., Blacker, T., Mitchell, S., "The Whisker-Weaving Algorithm: A Connectivity Based Method for Constructing All-Hexahedral Finite Element Meshes", Int. J. for Numer. Meth. in Eng., 39, 3327-3349, 1996.

[15] Canann, S.A., "Plastering and Optismoothing: New Approaches to Automated, 3D Hexahedral Mesh Generation and Mesh Smoothing", Ph.D. Dissertation, Brigham Young University, Provo, UT, 1991.

[16] Löhner, R., "Advances in unstructured mesh generation", Proc. ECCOMAS, 2000.

[17] Do Carmo, M.P., "Differential Geometry of Curves and Surfaces", Prentice Hall, 1976.

[18] Welch, W., Witkin, A., "Free-Form Shape Design Using Triangulated Surfaces”, In Compter Graphics (Proc. SIGGRAPH '94), 247-256, 1994.

[19] Merhof, D., Grosso, R., Tremel, U., Greiner, G., "Local Surface Approximation and its Application to Smoothing in Three-dimensional Indirect Mesh Generation", Technical Report Erlangen TR2/2004, FriedrichAlexander-University of Erlangen, 2004.

[20] Canann, S.A., Tristano, J.R., Staten, M.L., "An Approach to Combined Laplacian and Optimization-Based Smoothing for Triangular, Quadrilateral and Quad-Dominant Meshes", 7th International Meshing Roundtable, 479-494, 1998. 

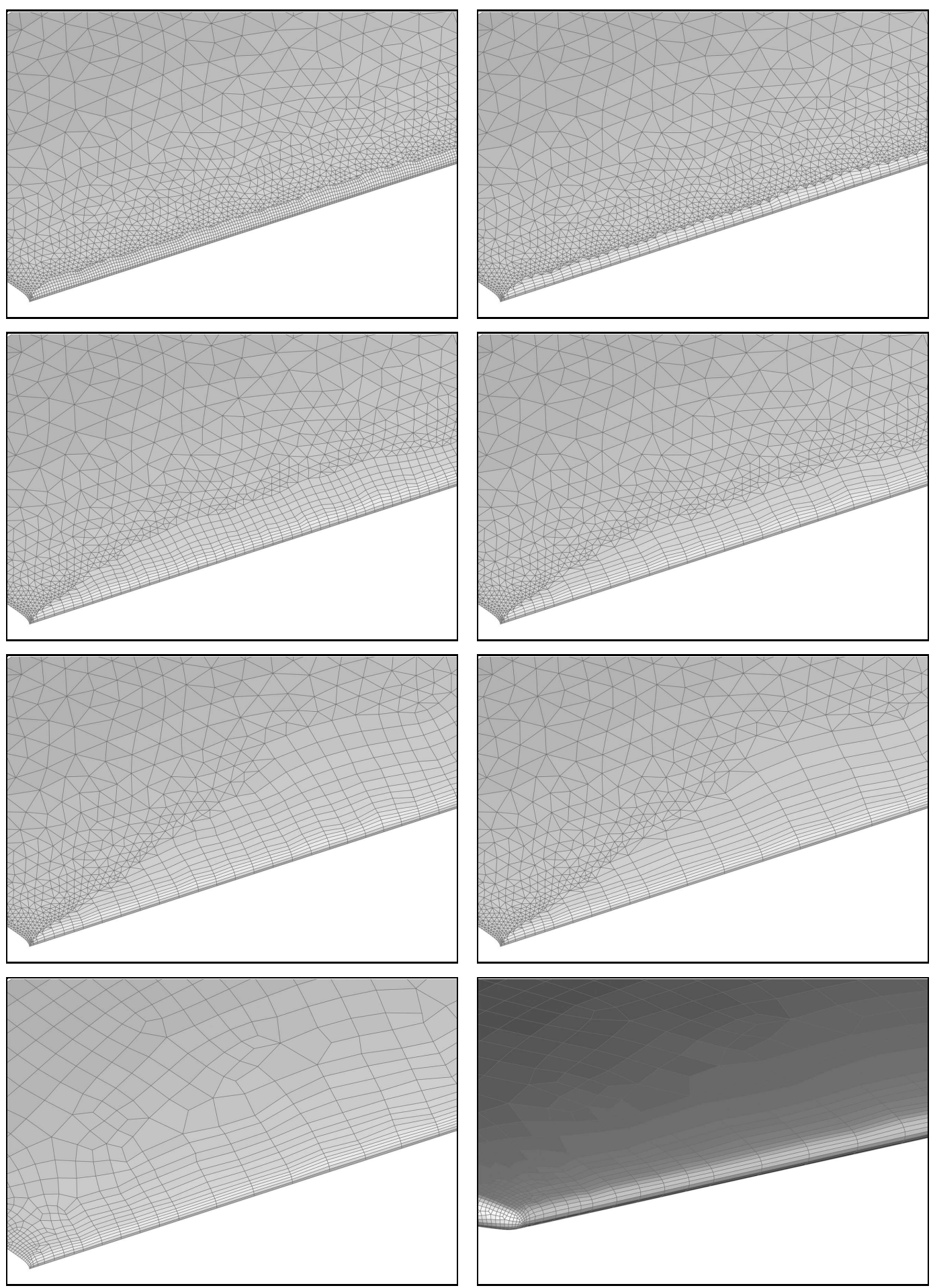

Fig. 7. Multi-Pass Three-Step-Approach. First to third row: Subsequent passes, for every pass the generation of structured rows of isotropic quadrilaterals is shown on the left, anisotropic merging is shown on the right. Last row (left): The remaining triangular mesh is filled with isotropic quadrilaterals. Last row (right): Completed air plane wing. Maximum anisotropy of 1:16 at leading edge. 